\title{
In-plane Displacement Measurement for Analytical Strain Determination
}

\author{
Alonso Saldaña-Heredia ${ }^{1 *}$, Pedro Antonio Márquez-Aguilar ${ }^{1}$ and José Antonio Vidal-Villegas ${ }^{2}$ \\ ${ }^{1}$ Center for Research in Engineering and Applied Sciences - ICBA, Universidad Autonoma del Estado de Morelos, Av. Universidad 1001, Col. Chamilpa. Cp. 62209, \\ Cuernavaca Morelos, Mexico \\ ${ }^{2}$ Center of Scientific Research and Higher Education of Ensenada, Baja California, Carretera Ensenada-Tijuana 3918, Col. Pedregal Playitas. Cp. 22860, Ensenada Baja \\ California, Mexico
}

\begin{abstract}
We present an optical experimental approach and an analytical model to calculate the strain on a brick when an external force-pulse is indirectly applied on its surface. We assumed that the displacement produced on the brick is energy that it transforms to deformation. To measure the displacement, we applied one laser-beam to the brick and took image data of the speckles created by its reflection. The speckles were processed using the digital image correlation technique and allowed us to evaluate the relative displacements caused on the surface of the brick. Then, based on the kinetic energy of forced and damped oscillations, we calculated analytical strain values of the order of $\varepsilon \approx 1.52 \times 10^{-6}$
\end{abstract}

Keywords: Laser speckle; In-plane displacement measurement; Digital image correlation; Force-pulse; Strain

\section{Introduction}

The strain measurement in materials is a relevant study as structures are involved in different stresses. It is well-known, from the stress-strain relation that every material eventually fails when it's close to the rupture part. Due to this possible consequence, the development of non-destructive strain measurement methods is a challenge for the assessment of structural elements in existing constructions.

Optical methods are an example of non-destructive methods, which have been used widely for measuring in-plane strain, stress, displacements, among others [1-3]. In the structural field, there is a method to measure structural displacement using a charge-coupleddevice camera plus digital signal processing [4]. Other methods are for instance the one developed by Jong-Woong et al. whom used two web cams to evaluate the horizontal displacement of high-rise buildings [5]; Hyoung-Suk et al. employed digital image processing, introducing a new dynamic vision system to measure displacements [6]; Sung-Wang and Nam-Sik [7] proposed digital image processing plus correlation to measure displacements in a structure model using a single point and multiple points.

As an alternative to measure displacements with a non-destructive technique, we implemented In-plane displacement measurements using Digital Image Correlation technique, which is able to measure an external force-pulse signal displacement and the strain caused by it. It is known that the simplest image matching procedure is Cross Correlation (CC) (which can be performed either in physical space $[8,9]$ or in Fourier space), the use of Fast Fourier Transform (FFT) helps to evaluate the CC function quickly [10]. Anuta [11] took advantage of the high speed of FFT algorithm doing digital multispectral and multitemporal statistical pattern-recognition. One of the advantage in doing Digital Image Correlation, is that users can create their own codes, as it is image processing, it is possible to process the images for a better result, such as selecting the best zone of interest.

To test our proposed method, we measured the displacement provoked by the propagation of an external force-pulse, using a laser beam focusing on the cross-section of a brick sample. The brick is a construction material made of backed clay, generally in a rectangular form. The material is in rest position and as the incoming force-pulse is propagated and strikes it, the brick will move according to the energy and will return to its initial, rest position.
The novelty in our proposed method is in coupling a displacement measurement to an analytical strain calculus. The main advantage is that this technique uses few optical components and is cheap for further applications: such as an optical displacement sensor.

It is well known that structures are under different kind of loads and also to external incoming-energies, thus it is important to have a nondestructive method to measure displacements and then calculate the strain. So, the aim of our study is to present a new alternative method to calculate the strain when the material is affected by an incoming force.

\section{Methodology}

\section{Laser speckle generation}

A laser beam propagates as a nearly unidirectional wave with little divergence and with finite cross-section [12]. The simplest beam and the most known is the Gaussian, because its characteristics and evolution are well-known [13]. The intensity distribution of a Gaussian beam is given by:

$$
I(r, z)=I_{0} \exp \left[\frac{-r^{2}}{\omega^{2}(z)}\right]
$$

where $I_{0}$ is the intensity of the beam at $z=0, r=\left(x^{2}+y^{2}\right)^{1 / 2}$ and $\omega(z)$ is the beam spot size at $z$ plane [14]. In the present work, the beam propagates through a lens with a focal length $f$ and is focused to the cross-section of a brick sample. To study this phenomenon, we need the Fresnel diffraction equation and the transformation due to the transmission through a lens [15] given by:

$$
U_{f}(u, v)=\frac{\exp \left[\frac{i k}{2 f}\left(u^{2}+v^{2}\right)\right]}{i \lambda f} \iint_{-\infty}^{\infty} U(x, y) \cdot t_{A} \cdot \exp \left[\frac{-i k}{f}(u x+v y)\right] d x d y
$$

where $U_{f}(u, v)$ is the distribution, $k$ is the wave number equal to $2^{\star} \pi$ /

*Corresponding author: Alonso Saldaña Heredia, Center for Research in Engineering and Applied Sciences-ICBA, Universidad Autonoma del Estado de Morelos, Av. Universidad 1001, Col. Chamilpa. Cp. 62209, Cuernavaca Morelos, Mexico, Tel: +52-7771376852; E-mail: alonso.saldana@uaem.mx

Received February 10, 2019; Accepted February 20, 2019; Published February 28,2019

Citation: Heredia AS, Aguilar PAM, Villegas JAV (2019) In-plane Displacement Measurement for Analytical Strain Determination. J Material Sci Eng 8: 510. doi: 10.4172/2169-0022.1000510

Copyright: (c) 2019 Heredia AS, et al. This is an open-access article distributed under the terms of the Creative Commons Attribution License, which permits unrestricted use, distribution, and reproduction in any medium, provided the original author and source are credited. 
$\lambda, \lambda$, is the wavelength of the laser beam, $f$ is the focal length of a lens and $u, v$ are the Fourier domain frequencies. The transmission through a lens $t_{A}$ is given by:

$$
t_{A}=\exp \left[\frac{i k}{2 f}\left(x^{2}+y^{2}\right)\right]
$$

and as our intensity is produced by a laser beam with an electromagnetic transverse mode $\mathrm{TEM}_{00}$ according to the manufacturer [16], we can write the signal distribution $U(x, y)$ as:

$$
U(x, y)=\exp \left[\frac{-\left(x^{2}+y^{2}\right)}{\omega^{2}}\right]
$$

Introducing equations (3) and (4) in (2), expanding formally and changing to polar coordinates we obtain:

$$
\begin{aligned}
& U(\rho)=\frac{\exp \left[C \rho^{2}\right]}{i \lambda} \\
& \text { where } \rho^{2}=u^{2}+v^{2}, \\
& C=\frac{i k}{2 f}+\frac{b^{2}}{4 a} \\
& \text { and } \\
& a=\frac{i k}{2 f}-\frac{1}{\omega^{2}} \\
& b=\frac{i k}{f}
\end{aligned}
$$

As equation (5) shows, we can assume that the intensity arrived at the focal length point is still a Gaussian distribution and as it impinges to a rough-like material, all the incoming light is transformed to random intensity distribution; thus, a speckle pattern is formed [17]. Those patterns are formed when coherent light is reflected from a rough surface or when light is propagated through a medium with random refractive index fluctuations [18].

In general the statistical properties of speckle patterns depend both on the coherence of the incident light and the detailed properties of the random surface or medium. Generally these kind of distributions are asymptotically Gaussian as it follows from the central limit theorem [19].

\section{Correlation technique}

Digital Image correlation (DIC), is an optical method that examines image data taken while samples of materials (bricks, metals, among others) are subjected to mechanical tests. This technique consists on capture consecutive images with a digital camera to evaluate the change in surface characteristics and understand the behavior of the material while it is subjected to an incoming force.

For correlation, it is necessary to obtain the spectrum between two images; let's assume that $A$ and $B$ are a pair of 2-D images, where $A$ represents the reference image and $B$ the displaced image [20]; therefore, it is taken the FFT for both images and the Cross-spectrum (Cs) is defined by:

$$
C_{S}(u, v)=F(u, v) \cdot G^{*}(u, v)
$$

where $F$ is the Fourier transform of the first image and $G^{*}$ is the complex-conjugate Fourier transform of the second image; once Cs is obtained, the cross-correlation (CC) is defined by:

$$
C_{C}=\frac{\operatorname{Re}\left(F^{-1}\left[C_{S}\right]\right)}{s_{1}^{2} s_{2}^{2} M}
$$

where $s_{1}^{2}$ and $s_{2}^{2}$ are the standard deviation of images $A$ and $B$, respectively and $M$ is the image size (in our case, we worked with $1080^{\star} 1080$ images). Thus, we normalize as we are assuming a totally Gaussian distribution in the speckle pattern.

\section{Theoretical analysis}

As we are indirectly applying force-pulses, we assume that the brick is affected first by the force impact, causing a peak amplitude, it can be modeled as a short time force oscillatory wave given by:

$$
f(t)=A_{p} \cdot \sin \left(w_{p} t-\alpha\right)
$$

where $A_{p}$ is the amplitude of $f(t), w_{p}$ and $\alpha$ are the frequency and the phase respectively [21].The structure will react to the force and return to its initial point according to damped oscillations:

$$
g(t)=A_{d} \cdot \exp ^{(-\gamma t)} \cdot \sin \left(w_{d} t-\alpha\right)
$$

where $A_{d}$ is the amplitude, $\Upsilon$ is the damping coefficient and $w_{d}$ is the frequency of the response [21]. In the present research, the brick sample is completely still at time $t=0$, the incoming force $P$ and the related energy $E_{p}$ would be set by $[21,22]$ :

$$
\begin{aligned}
& P=\sigma_{p} \cdot A_{i} \\
& E_{p}=P \cdot \vec{d}
\end{aligned}
$$

where $\sigma_{p}$ is the stress, $A_{i}$ is the impact area and $\vec{d}$ is the maximum displacement registered by DIC technique [22,23]. As the impact is along one axis of the plane, we can relate the total energy as a sum of both forced and damped Kinetic Energy waves:

$$
E_{k}=\frac{1}{2} \cdot m\left[f^{\prime}(t)^{2}+g^{\prime}(t)^{2}\right]=E_{k p}+E_{k d}
$$

Where $f^{\prime}(t)$ and $g^{\prime}(t)$ are the velocity obtained through deriving the signal, $E_{k p}$ and $E_{k d}$ are the Kinetic Energy of the forced and damped oscillations, respectively. The force impacts the surface of the table and the energy $E_{p}$ produced by this force is propagated through the brick. By using the energy law we can stablish that:

$$
E_{p}=E_{k}
$$

Substituting equations (13) and (14) in (16), we can obtain the strain provoked by the force by:

$$
\varepsilon=\frac{E_{k}}{E A_{i} \vec{d}}
$$

where $\varepsilon$ is the dimensionless strain and $E$ is the Young modulus of our material. We assume that every strain calculated remains in the elastic part of the stress-strain curve as the induced force is weak enough to provoke damage on the material.

\section{Experimental Set-up and Data Processing}

A table ( $\mathrm{T})$ is used to propagate four pulses to a brick with a Young's modulus of $15 \mathrm{GPa}$ [24]. The brick (Sa) was placed over the table and all pulses were induced through the longitudinal direction (Lon) of the brick. In Figure 1 is represented schematically our experimental set-up. The output of a Diode-Pumped Solid-State Laser (L) with a wavelength $\lambda=532 \mathrm{~nm}$ and power of $220 \mathrm{~mW}$ [16], is placed at $20 \mathrm{~cm}$ from the sample, the wave is propagated through a converging lens (l) with a focal distance of $f=7 \mathrm{~cm}$ which is placed in front of the sample (Sa) in order to irradiate the cross-section face and the scattering reflection impacts a screen $(\mathrm{Sc})$ which is placed in front of the sample at $15 \mathrm{~cm}$; the brick is first completely still, then the incoming force-pulse strikes horizontally to the table causing movement, while this action is taking place, the speckles generated are recorded with a Video Camera (VC). 
The video camera used for this experiment can record in a rate of 240 frames per second with a 1980x1080 image resolution. Once the video was recorded, we used Video to JPG free software [25]; this software enables the user to split the video into each possible frame. We wrote a program in Matlab for doing Digital Image Correlation (DIC) in which we load and process all the frames. The image processing is depicted in the following images: In Figure 2a it is shown how the code loads the image and then it is converted into a gray-scale image (Figure 2b). Therefore the image is crop into $1080 \times 1080$ to begin the FFT analysis of all depicted images (Figure 2c), taking the first as the reference and the others are displaced images.

\section{Results and Discussions}

As a forementioned, we indirectly applied external force-pulses to a brick, and measured the induced displacement by means of in-plane DIC; from this we got an analytical approach of the strain provoked by the induced pulse. All incoming force-pulses are studied in the focal plane of the lens and along to the longitudinal direction of the brick. In Figures 3-7 we show the obtained results.

Figure 3 shows the result of the first test: in (a) We can see the response of the system table-brick to the induced force-pulse. We can see in (b) the signal obtained from DIC technique, shows a 23 $\mathrm{mm}$ maximum amplitude, the decay of the recorded pulse last $10 \mathrm{sec}$ approximately. Figure $3 \mathrm{~b}$ is the analytical approach to the movement
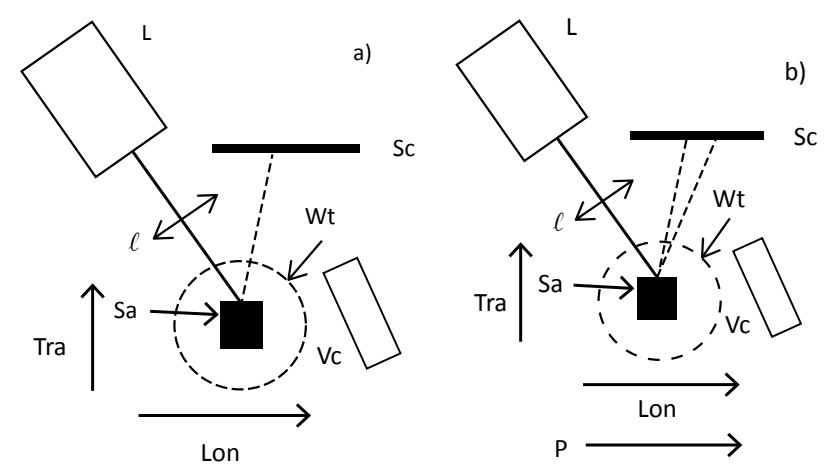

Figure 1: Experimental set-up: (a) Brick totally still at $t=0$; (b) Movement registration when the force-pulse is applied.
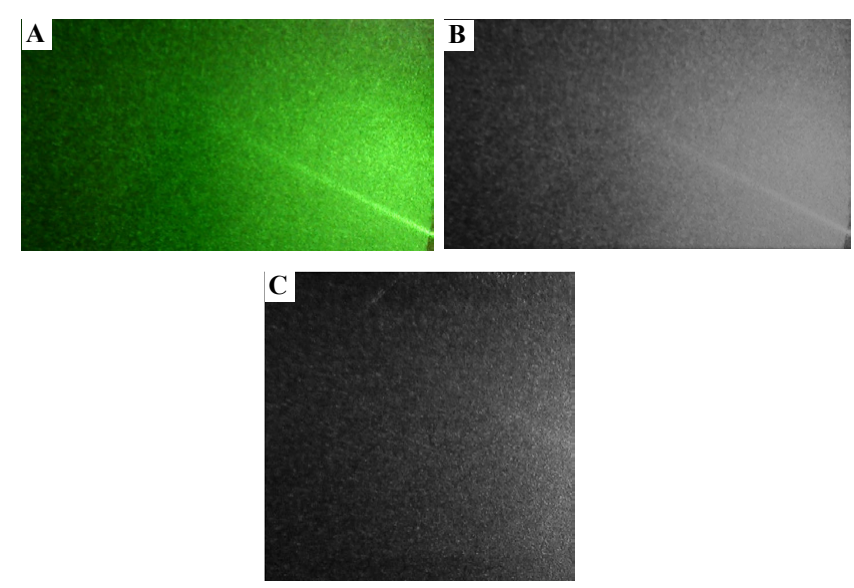

Figure 2: Image processing: (a) Speckle reflection; (b) Gray-scale image; (c) 1080 Square crop.

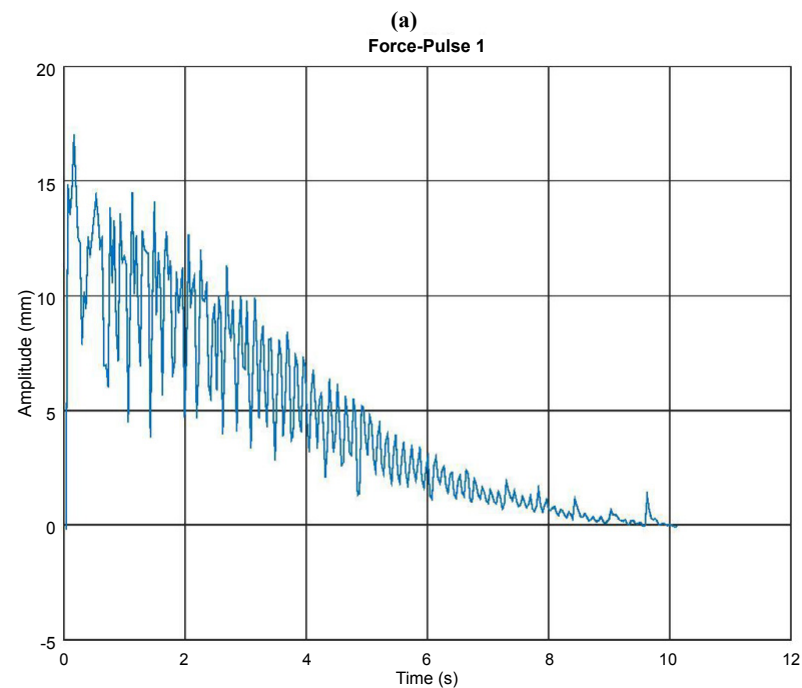

(b)

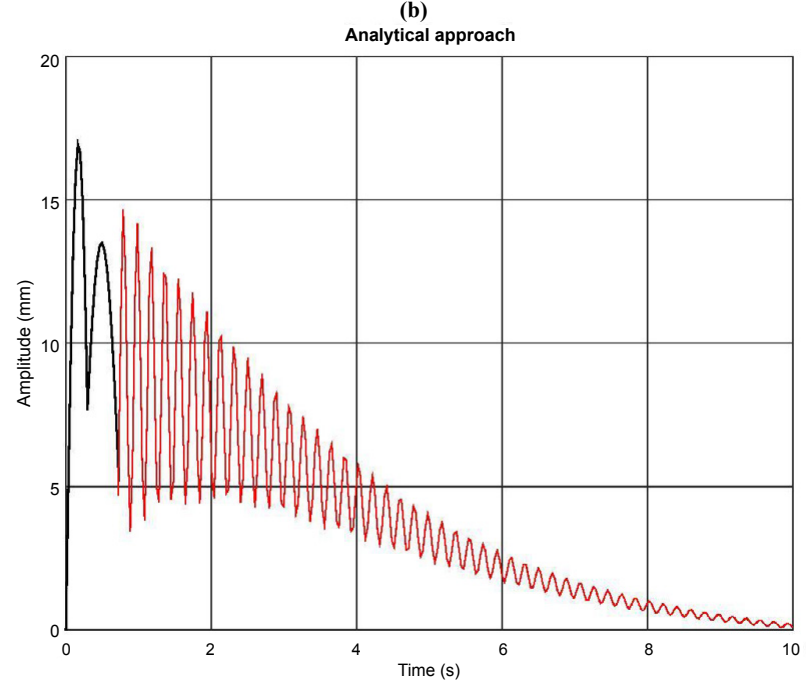

Figure 3: Test 1 (a) displacement obtained by DIC (b) Analytical approach.

according to Equations (11) (black plot) and (12) (red plot), the values used to do the analytical approach are in Table 1. In Figure 4 we show the comparison between the displacement measured and the analytical approach.

In this first test, the analytical approach has a correlation coefficient of 0.83 and has a mean accuracy of $94 \%$ from the digital signal acquired. The accuracy has obtained by calculating first the relative error:

$$
e_{r r}=\frac{V_{r}-V_{e}}{V_{r}}
$$

In which $V_{r}$ and $V_{e}$ are the real and experimental data. Once calculated a mean error we can obtain the mean accuracy by doing 1- $e_{r r}$. Next, we present the results derived from the application of another three pulses to the system table-brick.

In the comparison of the fourth tests, we can observe that the analytical model fits to the signal acquired by in-plane DIC, the mean correlation coefficient for them were 0.8 , which is a good approximation to the real signal. Residual graphs were obtained by subtracting the analytical approach from the experimental data obtained by DIC, in them, we can observe that for the first 6 sec we have the most differences, 


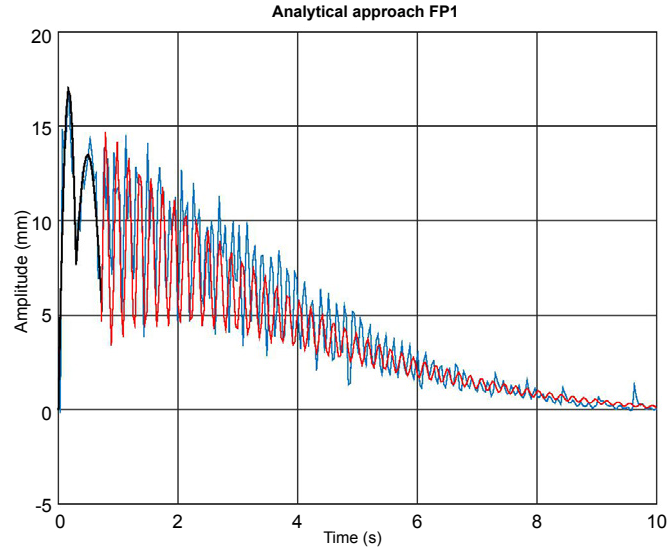

(a)

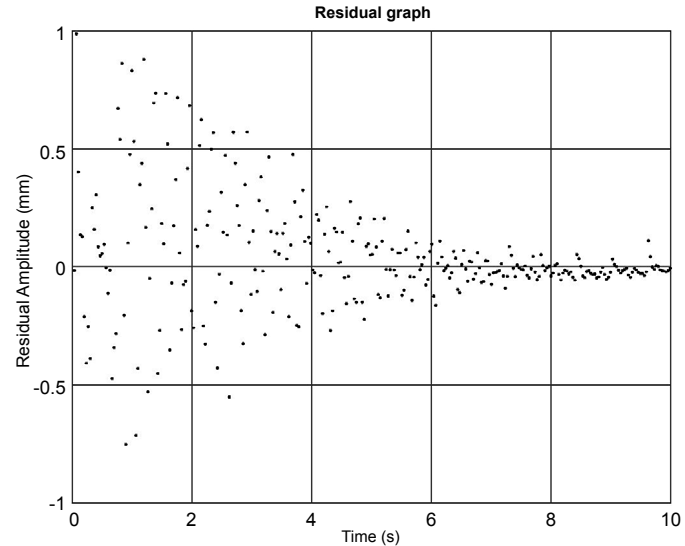

(b)

Figure 4: Comparison for the first test: (a) In-plane displacement (blue) and analytical approach (black-red); (b) Residual graph.

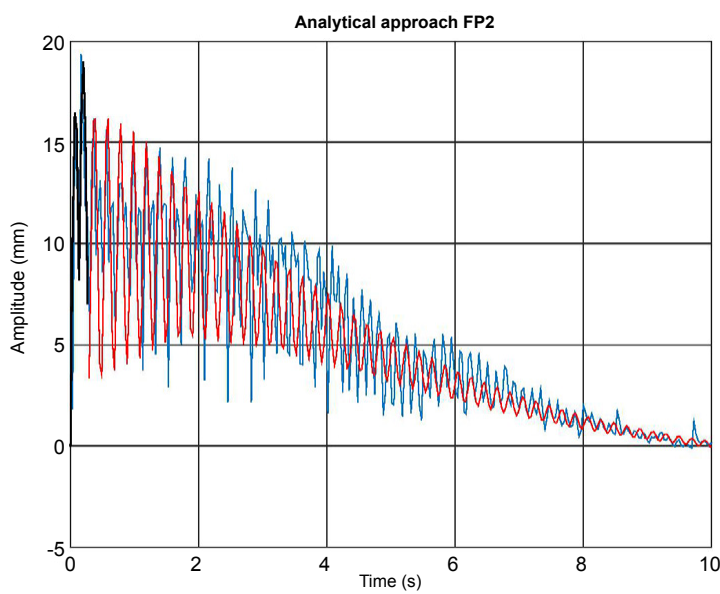

(a)

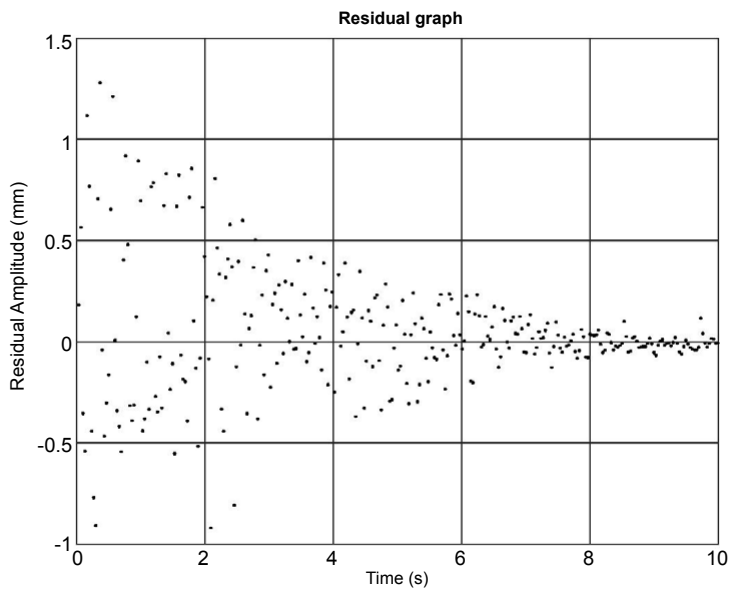

(b)

Figure 5: Comparison for the second test: (a) In-plane displacement (blue) and analytical approach (black-red); (b) Residual graph.

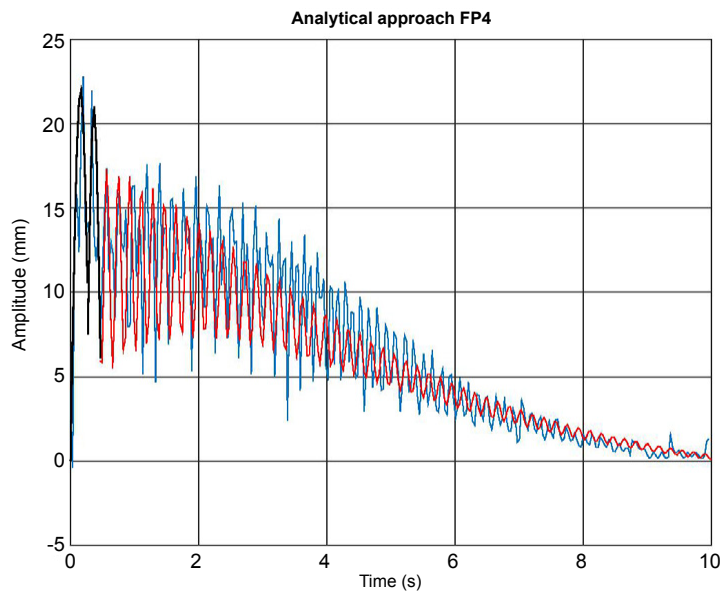

(a)

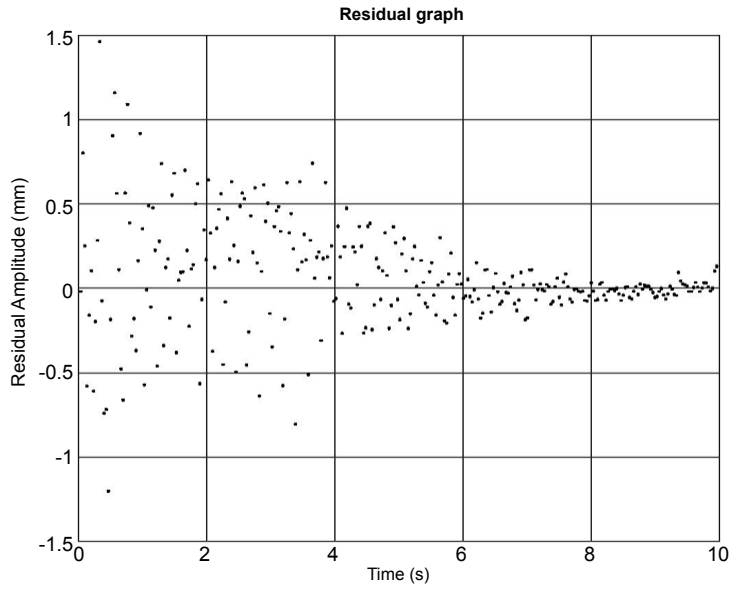

(b)

Figure 6: Comparison for the third test: (a) In-plane displacement (blue) and analytical approach (black-red); (b) Residual graph. 


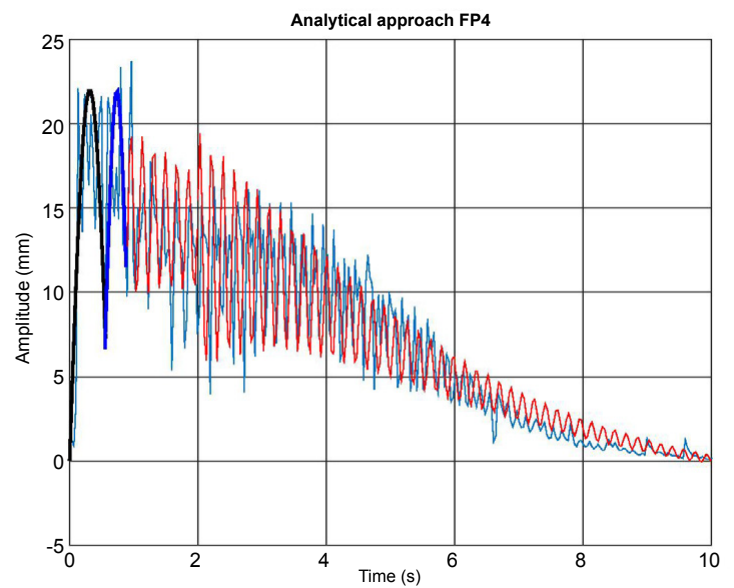

(a)

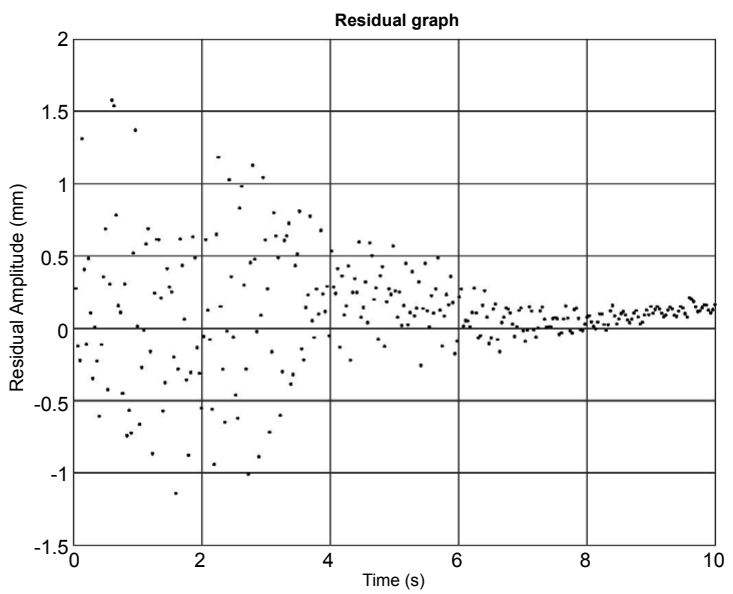

(b)

Figure 7: Comparison for the fourth test: (a) In-plane displacement (blue) and analytical approach (black-red); (b) Residual graph.

it is mainly because the analytical model follows a prescribed equation and the signal we acquire has fluctuations in time as a natural reaction to the induced force-pulse; in the interval time of 6 to $10 \mathrm{sec}$, we can observe that the analytical model has a better fit, giving us differences lower than 0.3 .

The analytical approach were done by using equations (11) and (12), where the data used for them are described in Table 1.

The data of this table were obtained by means of fitting iteratively the values in the equations (11) and (12). We see that the analytical data used for the tests are almost the same values. We observe that every test has an analytical response with high frequency and the initial amplitude just varied $5 \mathrm{~mm}$.

The strain values are calculated by the analytical model proposed in equation (17) and the data used are shown in Table 2.

In Table 2, we have a total Kinetic Energy $E_{k}$ which is the sum of $E_{k p}$ and $E_{k d}$ as we stablished in the previous section, also it is seen that the impact area $A_{i}$ has the same value, it is because we used the same object to induce the force-pulses, thus we measure its impact area. Third test has a particular behavior, comparing with first test, the amplitude $A_{d}$ registered was higher but the strain was lower, according to Equation (17) in which is stablished that strain is inversely proportional to the maximum displacement registered $\vec{d}$.

As a summary, in Table 3 we show the number of the applied pulses, the related Kinetic Energy, the strain caused on the brick and the accuracy of the tests.

From Table 3, we can see that this technique allows to measure strain indirectly with a mean value of $1.528 \times 10^{-6}$. Also it is seen that there is a relation between energy and strain provoked; in pulses 1 and 3 we can see that there is almost the same total Kinetic Energy compared with pulse 2 and the strain varies in the second test about $0.1 \times 10^{-6}$; for the third test it has a higher strain variation compared with pulses 1 and 2 and we referred it due to the maximum displacement registered in the signal. If we compare fourth test with the first one, we can see about $0.15 \mathrm{~J}$ more but the strain caused by it is just $0.1 \times 10^{-6}$ greater.

Some other methodologies are used to obtain low values of strain such as strain gages, which can get values of strain in the micrometer

\begin{tabular}{|c|c|c|c|c|c|c|c|}
\hline Test & $\boldsymbol{A}_{\boldsymbol{p}}(\boldsymbol{m})$ & $\begin{array}{c}\boldsymbol{W}_{\boldsymbol{p}} \\
(\mathbf{r a d} / \boldsymbol{s})\end{array}$ & $\boldsymbol{E}_{\boldsymbol{k} \boldsymbol{p}}(\boldsymbol{J})$ & $\boldsymbol{A}_{\boldsymbol{d}}(\boldsymbol{m})$ & $\begin{array}{c}\boldsymbol{W}_{\boldsymbol{d}} \\
(\mathbf{r a d} / \mathbf{s})\end{array}$ & $\boldsymbol{V ( 1 / s )}$ & $\boldsymbol{E}_{\boldsymbol{k d}}(\boldsymbol{J})$ \\
\hline 1 & 0.017 & 9 & 0.0754 & 0.003 & 33 & 0.47 & 0.33471 \\
\hline 2 & 0.017 & 10 & 0.0139 & 0.003 & 31 & 0.4 & 0.3703 \\
\hline 3 & 0.022 & 10 & 0.0527 & 0.0035 & 35 & 0.4 & 0.3535 \\
\hline 4 & 0.022 & 10 & 0.023 & 0.0015 & 35 & 0.4 & 0.5411 \\
\hline
\end{tabular}

Table 1: Data used in Eq. (11) and (12) to compute the analytical model.

\begin{tabular}{|c|c|c|c|c|}
\hline Test & $\boldsymbol{E}_{\boldsymbol{k}}(\boldsymbol{J})$ & $\boldsymbol{A}_{\boldsymbol{i}}\left(\boldsymbol{m}^{2}\right)$ & $\overrightarrow{\boldsymbol{d}}(\boldsymbol{m})$ & $\boldsymbol{\varepsilon}(\boldsymbol{m} / \boldsymbol{m})$ \\
\hline 1 & 0.4101 & $1 \times 10^{-5}$ & 0.017 & $1.608 \times 10^{-6}$ \\
\hline 2 & 0.3843 & $1 \times 10^{-5}$ & 0.017 & $1.507 \times 10^{-6}$ \\
\hline 3 & 0.4062 & $1 \times 10^{-5}$ & 0.018 & $1.5045 \times 10^{-6}$ \\
\hline 4 & 0.5641 & $1 \times 10^{-5}$ & 0.022 & $1.709 \times 10^{-6}$ \\
\hline
\end{tabular}

Table 2: Data used in Eq. (17) to compute the analytical strain.

\begin{tabular}{|c|c|c|c|}
\hline Force pulse & $\boldsymbol{E}_{\boldsymbol{k}}(\boldsymbol{J})$ & $\boldsymbol{\varepsilon}(\boldsymbol{m} / \mathbf{m})$ & Accuracy \\
\hline 1 & 0.4101 & $1.608 \times 10^{-6}$ & $94 \%$ \\
\hline 2 & 0.3843 & $1.507 \times 10^{-6}$ & $90 \%$ \\
\hline 3 & 0.4062 & $1.5045 \times 10^{-6}$ & $93 \%$ \\
\hline 4 & 0.5641 & $1.709 \times 10^{-6}$ & $92 \%$ \\
\hline
\end{tabular}

Table 3: Strain caused on the brick by the energy generated from the force-pulse tests.

region [26,27]. Optical methods such as Interferometry used with a wavelength of $0.51 \mu \mathrm{m}$ can generate a resolution of $0.047 \mu \mathrm{m}$ [28]. A micro Moiré Interferometry can obtain values of strain of 0.0036 [29]. Another optical method stablish that with beam propagation the user can obtain values in the order of $1 \times 10^{-4}[30,31]$.

The present technique helps us to obtain values of strain when an incoming force in the range of $1.9-2.5 \mathrm{kN}$ is affecting a sample, it can be mentioned that is a novel procedure. Our proposed technique can be improved by doing standardized force-pulse test, as we see in the results, each signal obtained from the force-pulses was different one from the other and also it can be improved by doing standardized mechanical impact test.

Some of the advantages of this technique are that we can measure indirectly the strain by means of Digital Image Correlation; while the sample is subjected to an induced force movement. With this strain measured, we are able to know a better total stress concentration in a 
selected zone [22]. A disadvantage is that in our experimental setup, we use a lens which must be in the focal length distance, if the induced force movement displace the lens, our experiment will experience some trouble in image processing.

\section{Conclusions}

We proposed a methodology to analytically calculate the strain on a sample of brick by force-pulses applied indirectly to it. The proposed optical method based on the use of laser speckles and digital image correlation can be used to determine the strain on a material in the micrometric order: $\varepsilon \approx 1.52 \mu \mathrm{m}$ with a correlation coefficient of 0.8 and an accuracy of $92 \%$. This methodology is inexpensive and nondestructive; however, it must be improved by standardizing the forcepulse test.

\section{Acknowledgements}

Alonso Saldaña Heredia thanks to National Council of Science and Technology of Mexico (CONACYT) for the grant No. 360140. Thanks to CICESE for accepting me during a six-month doctoral stay. And, we appreciate the comments to the manuscript by professor $\mathrm{S}$. Mahmoud Abdalla.

\section{References}

1. Pradille C, Bellet M, Chastel Y (2010) A Laser speckle method for measuring displacement field. Application to resistance heating tensile test on steel. Applied Mechanics and Materials 24: 135-140.

2. Stanciu $A$, Cotoros $D$, Baritz $M$, Rogozea $L$ (2010) Analysis by strain gauges of the strains in a composite material. Proceedings of the 9th WSEAS International Conference on Signal Processing, Robotics and Automation, UK.

3. Solle J, Linares JM, Sprauel JM, Mermoz E (2012) Optical measurement for the estimation of contact pressure and stress. CIRP Annals Manufacturing Technology 61: 483-486.

4. Gongkang F, Adil GM (2002) An optical approach to structural displacement measurement and its application. J Eng Mech 128: 511-520.

5. Jong-Woong P, Jong-Jae L, Hyung-Jo J, Hyun M (2010) Vision-based displacement measurement method for high-rise building structures using partitioning approach. NDT\&E International 43: 642-647.

6. Hyoung-Suk C, Jin-Hwan C, Sang-Hyo J, Jin-Hee A (2011) Structural dynamic displacement vision system using digital image processing. NDT\&E International 44: 597-608.

7. Sung-Wan K, Nam-Sik K (2011) Multi-point displacement response measurement of civil infrastructures using digital image processing. Procedia Engineering 14: 195-203.

8. Peters WH, Ranson WF (1982) Digital image techniques in experimental stress analysis. Opt Eng 21: 427-441.

9. Sutton MA, Mc Neill SR, Helm JP, Chao YJ (2000) Advances in two dimensional and three dimensional computer vision. Photomechanics, Topics in Applied Physics 77, Springer, Berlin: 323-372.

10. Chen DJ, Chiang FP, Tan YS, Don HS (1993) Digital speckle-displacement measurement using a complex spectrum method. Appl Opt 32: 1839-1852.
11. Anuta PE (1970) Spatial registration of multispectral and multitemporal digital imagery using fast Fourier transform techniques. IEEE Trans Geosci Electron 8: 353-368.

12. Sirohi RS (2009) Optical methods of measurement: Wholefield techniques. Taylor and Francis Group, US, pp: 316.

13. Alda J (2003) Laser and gaussian beam propagation and transformation. Encyclopaedia of optical engineering, Marcel Dekker Inc, pp: 999-1013.

14. Yariv A (1990) Quantum Electronics. (3rd Edn), John Wiley \& Sons, USA, pp: 693.

15. Goodman JW (2005) Introduction to Fourier Optics, (2nd edn), McGraw Hill Companies, US, pp: 457.

16. Laserglow Technologies (2015) LCS-0532 Low-Cost DPSS Laser System Laserglow Part Number: C53200XSX. Laser Product Datasheet generated on 2015.

17. Dainty JC (1975) Laser speckle and related phenomena. Springer-Verlag Berlin Heidelberg, New York, 9: 295.

18. Born M, Wolf E (1970) Principles of optics. (4th edn), Pergamon Press, UK, pp: 859.

19. Goodman JW (1976) Some fundamental properties of speckle. J Opt Soc Am 66: 1145-1150.

20. Sutton MA, Orteu JJ, Schreirer HW (2009) Image Correlation for Shape, Motion and Deformation Measurements: Basic Concepts, Theory and Applications. Springer, US, pp: 328.

21. Serway RA, Jewett JW, Hernández AEG, López EF (2002) Física para cienciase ingeniería (No. 530530 S4F5 2002 S47F5 2002). McGraw-Hill.

22. Gere J, Goodno B (2009) Mecánica de Materiales. (7th Edn), Cengage Learning Editores, S.A. MÃ@xico, D.F. pp: 1029.

23. Beer FP, Johnston ER, DeWolf JT, Mazurek DF (2012) Mechanics of Materials. (6th Edn), McGraw Hill, New York, pp: 838.

24. Saldaña HA, Márquez APA, Molina OA, Zamudio LA (2015) Digital image correlation used to analyze a brick under compression test. Proceedings Volume 9660, SPECKLE 2015: VI International Conference on Speckle Metrology; 96600R.

25. https://free-video-to-jpg-converter.uptodown.com/windows

26. Hidalgo CF (1995) Analisis experimental de esfuerzos aplicaciones con galgas extensometricas. Tesis de Maestria. Universidad Autonoma de Nuevo Leon.

27. Dally JW, Riey WF (1991) Experimental Stress Analysis. (3rd Edn), McGrawHill Inc, New York, pp: 990.

28. Chen F, Brown GM, Song M (2000) Overview of three-dimmensional shape measurement using optical methods. Opt Eng 389: 10-22.

29. Asundi AK (2009) MoirÃ@ Interferometric Strain Sensor (MISS) for Deformation and Strain Measurement. School of Mechanical \& Production Engineering, Nanyang Technological University, Singapore, Experimental Techniques, pp: $162-167$.

30. Saldaña HA, Márquez APA, Molina OA (2015) Concrete Stress-Strain Characterization by Digital Image Correlation. J Appl Mech Eng 4: 189.

31. Saldaña HA, Márquez APA, Molina OA (2016) Stress-Strain Characterization in Fiber-Reinforced Composites by Digital Image Correlation. J Material Sci Eng 5: 286. 